\title{
A quadratic upper bound on the size of a synchronizing word in one-cluster automata.
}

\author{
Marie-Pierre Béal ${ }^{1}$ and Dominique Perrin ${ }^{1}$ \\ Université Paris-Est, LIGM CNRS, 77454 Marne-la-Vallée Cedex 2, France \\ \{beal, perrin\}@univ-mlv.fr
}

\begin{abstract}
. Černy's conjecture asserts the existence of a synchronizing word of length at most $(n-1)^{2}$ for any synchronized $n$-state deterministic automaton. We prove a quadratic upper bound on the length of a synchronizing word for any synchronized $n$-state deterministic automaton satisfying the following additional property: there is a letter $a$ such that for any pair of states $p, q$, one has $p \cdot a^{r}=q \cdot a^{s}$ for some integers $r, s$ (for a state $p$ and a word $w$, we denote by $p \cdot w$ the state reached from $p$ by the path labeled $w$ ). As a consequence, we show that for any finite synchronized prefix code with an $n$-state decoder, there is a synchronizing word of length $O\left(n^{2}\right)$. This applies in particular to Huffman codes.
\end{abstract}

\section{Introduction}

Synchronized automata are deterministic and complete finite-state automata admitting a synchronizing word, that is a word which takes each state of the automaton to a single special state. Cerný conjecture claims that each $n$-state synchronized automaton has a synchronizing word of length at most $(n-1)^{2}$ [5]. An extension of this conjecture due to Pin $[12,14]$ was shown to be false by Kari [9]. The conjecture has been shown to be true for particular classes of automata like the class of circular automata by Dubuc [6] (see also [13]). A $n(n-1) / 2$ upper bound has been obtained by Trahtman $[17,19]$ for aperiodic automata. This upper bound was improved to $n(n+1) / 6$ by Volkov [20] (see also [21]).

In a previous note [1], the first author gave a proof of a quadratic bound for circular automata which is simpler than the one given in [6]. Nevertheless, it does not allow one to get the tight $(n-1)^{2}$ bound. The proof uses rational series.

The formulation of the problem in terms of rational series is also used in [1] to provide a simple proof of a result from Kari [10] which proves Černý's conjecture for automata with an underlying Eulerian graph.

Later, the result on circular automata was generalized by Carpi and d'Alessandro to a larger class called strongly transitive automata [4]. 
Their proof uses rational series as in [1]. They use the same methods to generalize the result of Kari to unambiguous automata.

In this paper, we prove the existence of a quadratic upper bound for the length of a synchronizing word for a class of finite automata called one-cluster. This means that, for some letter $a$, there is only one cycle with all edges labeled by $a$. The proof is an extension of the argument of [1] and uses again rational series.

The class of one-cluster automata contains in particular the automata associated with finite prefix codes. We thus obtain the existence of a quadratic bound on the length of a synchronizing word for a finite maximal synchronized prefix code. This applies in particular to Huffman codes.

Let us mention two recent results connected to our work (we thank Hugo Vaccaro for pointing out these references to us). First, it is proved in [8] that almost all finite maximal prefix codes are synchronizing. Next, in [3], it is proved that a finite maximal synchronized prefix code with $n$ codewords of maximal length $h$ has a synchronizing word of length $O(n h \log n)$. This bound is not comparable with ours. Indeed, since $\log n \leq$ $h \leq n-1$, one has $n(\log n)^{2} \leq n h \log n \leq n^{2} \log n$.

\section{Automata and series}

Let $A$ be a finite alphabet and $A^{*}$ be the set of finite words drawn from the alphabet $A$, the empty word $\epsilon$ included. A (finite) automaton $\mathcal{A}$ over some (finite) alphabet $A$ is composed of a finite set $Q$ of states and a finite set $E$ of edges which are triples $(p, a, q)$ where $p, q$ are states and $a$ is a symbol from $A$ called the label of the edge. Note that we do not specify a set of terminal states and that, for this reason, our automata are sometimes called semi-automata.

An automaton is deterministic if, for each state $p$ and each letter $a$, there is at most one edge starting in $p$ and labeled with $a$. It is complete deterministic if, for each state $p$ and each letter $a$, there is exactly one edge starting in $p$ and labeled with $a$. This implies that, for each state $p$ and each word $w$, there is exactly one path starting in $p$ and labeled with $w$. We denote by $p \cdot w$ the state which is the end of this unique path.

An automaton is irreducible if its underlying graph is strongly connected.

A synchronizing word of a deterministic complete automaton is a word $w$ such that for any states $p, q$, one has $p \cdot w=q \cdot w$. A synchronizing word is also called a reset sequence or a magic sequence, or also a homing word. 
An automaton which has a synchronizing word is called synchronized (see an example on the right part of Figure 1).
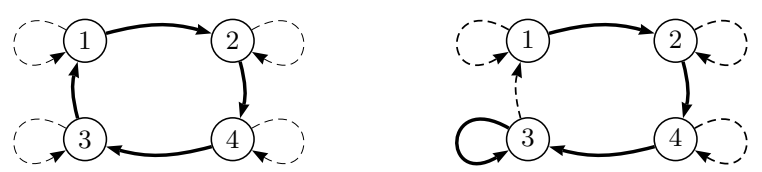

Fig. 1. Two complete deterministic automata labeled on $A=\{a, b\}$. A thick plain edge is an edge labeled by $a$ while a thin dashed edge is an edge labeled by $b$. The automaton on the left is not synchronized. The one on the right is synchronized; for instance, the word $a a a$ is a synchronizing word.

Let $\mathcal{A}=(Q, E)$ be a complete deterministic automaton. For any word $u \in A^{*}$, we denote by $M_{u}$ the transition matrix of the action of $u$ on the states $Q$. It is defined by:

$$
\left(M_{u}\right)_{p q}= \begin{cases}1 & \text { if } p \cdot u=q \\ 0 & \text { otherwise }\end{cases}
$$

Note that if $u, v$ are two words, we have

$$
M_{u v}=M_{u} M_{v} .
$$

We define the rank of a word $u$ as the cardinality of $Q \cdot u$. Note that since the automaton is complete deterministic, this rank is non null, and that a word is synchronizing if and only if his rank is 1 .

A circular automaton is a deterministic complete automaton on the alphabet $A$ such that there is a letter $a$ of $A$ which induces a circular permutation of the states, i.e. such that $M_{a}$ is a circular permutation matrix.

We shall consider the set of non commutative formal series with coefficients in a ring $K$ (with $K=\mathbb{Z}$ or $K=\mathbb{Q}$ ), which are applications from $A^{*}$ to $K$. If $S$ is such a series, the image of a word $u$ of $A^{*}$ by $S$ is denoted by $\langle S, u\rangle$ and called the coefficient of $u$ in $S$.

As an example, the series $S$ on $\{a, b\}^{*}$ with coefficients in $\mathbb{Z}$ defined by $\langle S, u\rangle=|u|_{a}-|u|_{b}$ maps a word $u \in\{a, b\}^{*}$ to the difference between the number of occurrences of $a$ and $b$ in $u$.

A $K$-linear representation of dimension $d$ of a series $S$ is a triple $(\lambda, \mu, \gamma)$ where $\lambda \in K^{1 \times d}, \mu$ is a morphism from $A^{*}$ to $K^{d \times d}$, and $\lambda \in$ $K^{d \times 1}$, such that

$$
\langle S, u\rangle=\lambda \mu(u) \gamma
$$


A series $s$ is $K$-rational if it has a $K$-linear representation. Its rank on $K$ is the minimal dimension of all its linear representations.

For example, the series $S$ defined on $\{a, b\}^{*}$ by $\langle S, u\rangle=|u|_{a}-|u|_{b}$ is rational of rank 2 . The triple $(\lambda, \mu, \gamma)$ defined by

$$
\lambda=\left[\begin{array}{ll}
1 & 0
\end{array}\right], \mu(a)=\left[\begin{array}{ll}
1 & 1 \\
0 & 1
\end{array}\right], \mu(b)=\left[\begin{array}{cc}
1 & -1 \\
0 & 1
\end{array}\right], \gamma=\left[\begin{array}{l}
1 \\
0
\end{array}\right]
$$

is a $\mathbb{Z}$-linear representation of $S$ of dimension 2 .

Cerný's conjecture gives an upper bound on the size of a shortest synchronizing word in a synchronized automaton.

Conjecture 1 (Černý 1964). A synchronized $n$-state deterministic complete automaton has a synchronizing word of length at most $(n-1)^{2}$.

The conjecture was proved by Dubuc for circular automata.

Proposition 1 (Dubuc 1998). A circular synchronized n-state deterministic complete automaton has a synchronizing word of size at most $(n-1)^{2}$.

\section{One-cluster automata}

In the sequel $\mathcal{A}=(Q, E)$ denotes an $n$-state deterministic and complete automaton over an alphabet $A$. We fix a particular letter $a \in A$.

Let $\mathcal{R}$ be the subgraph of the graph of $\mathcal{A}$ made of the edges labeled by $a$. The graph $\mathcal{R}$ is a disjoint union of connected component called $a$-clusters. Since each state has exactly one outgoing edge in $\mathcal{R}$, each $a$ cluster contains a unique cycle, called an $a$-cycle, with trees attached to the cycle at their root. For each state $p$ of the $a$-cluster, we define the level of $p$ as the distance between $p$ and the root of the tree containing $p$. If $p$ belongs to the cycle, its level is null. The level of the automaton is the maximal level of its states.

A one-cluster automaton with respect to a letter $a$ is a complete deterministic automaton which has only one $a$-cluster. Equivalently, an automaton is one-cluster if it satisfies the following condition: for any pair of states $p, q$, one has $p \cdot a^{r}=q \cdot a^{s}$ for some integers $r, s$.

Note that a one-cluster automaton whose level is null is circular.

Let $C$ be a cycle of $\mathcal{A}$ and $P$ be a subset of $C$. A word $u$ is said to be $P$-augmenting if

$$
\operatorname{card}\left(P u^{-1} \cap C\right)>\operatorname{card}(P),
$$


where we denote $P u^{-1}=\{q \in Q \mid q \cdot u \in P\}$.

We now prove the existence of a quadratic upper bound on the size of a shortest synchronizing word in a synchronized automaton.

Proposition 2. Let $\mathcal{A}$ be a synchronized n-state deterministic complete automaton. If $\mathcal{A}$ is one-cluster, then it has a synchronizing word of length at most $1+2(n-1)(n-2)$.

We prove the proposition for irreducible automata. The case of reducible automata easily reduces to this one. Let $\mathcal{A}=(Q, E)$ be a deterministic complete and irreducible $n$-state automaton.

Since Cerný's conjecture is proved for circular automata, we may assume that the level $\ell$ of the automaton is greater than or equal to 1 .

Let $C$ be the $a$-cycle and let $m$ be the length of $C$. Let $P$ be a subset of $C$. Note that a word $u$ is a $P$-augmenting word if and only if

$$
C M_{u} P^{t}>C P^{t}
$$

where $P, C$ denote the characteristic row vectors of the sets $P, C$. Indeed,

$$
C M_{u} P^{t}=\sum_{r \in C, s \in P}\left(M_{u}\right)_{r s}=\operatorname{card}\{r \in C \mid r \cdot u \in P\}=\operatorname{card}\left(P u^{-1} \cap C\right) .
$$

Similarly, $C P^{t}=\operatorname{card}(P)$.

We denote by $S$ the series defined by:

$$
\langle S, u\rangle=C M_{u} P^{t}-C P^{t},
$$

By definition, one has $\langle S, u\rangle>0$ if and only if $u$ is a $P$-augmenting word.

Lemma 1. The series $S$ has rank on $\mathbb{Q}$ at most $n$.

Proof. The series $S$ is $\mathbb{Z}$-rational since it is the difference of two $\mathbb{Z}$-rational series (the second one is actually a constant). It has the following linear representation $(\lambda, \mu, \gamma)$ with $\lambda \in \mathbb{Z}^{1 \times 2 n}, \mu: A^{*} \rightarrow \mathbb{Z}^{2 n \times 2 n}, \lambda \in \mathbb{Z}^{2 n \times 1}$,

$$
\lambda=(C,-C), \mu(u)=\left[\begin{array}{cc}
M_{u} & 0 \\
0 & I
\end{array}\right], \gamma=\left[\begin{array}{l}
P^{t} \\
P^{t}
\end{array}\right],
$$

since $\langle S, u\rangle=\lambda \mu(u) \gamma$. The rank of $S$ on $\mathbb{Q}$ is bounded above by the dimension of the $\mathbb{Q}$-vector space generated by the row vectors $\left(C M_{u},-C\right)$. This space is included in the vector space generated by the vectors $\left(C M_{u}-\right.$ $C, \mathbf{0})$ and the row vector $(C,-C)$, where $\mathbf{0}$ is the null column vector of size $n$. Thus the rank of $S$ is at most equal to the dimension of the vector 
space $V$ generated by the vectors $C\left(M_{u}-I\right)$, plus one. We now show that the dimension of $V$ is at most $n-1$. Since the automaton $\mathcal{A}$ is complete deterministic, for any $u \in A^{*}, M_{u} \mathbf{1}=\mathbf{1}$, where $\mathbf{1}$ is the column vector with coefficients 1 of size $n$. This implies that $C\left(M_{u}-I\right) \cdot \mathbf{1}=0$. Thus the vectors of $V$ are orthogonal to the vector $\mathbf{1}$. The dimension of $V$ is thus at most $n-1$. This proves that the rank of $S$ on $\mathbb{Q}$ is at most $n$.

We denote by $T$ the $\mathbb{Z}$-rational series defined by

$$
\langle T, u\rangle=\left\langle S, u a^{\ell}\right\rangle
$$

where $\ell$ denotes the level of the automaton $\mathcal{A}$. If $(\lambda, \mu, \gamma)$ is a $\mathbb{Q}$-linear representation of $S$ of dimension $n$, then $\left(\lambda, \mu, \mu\left(a^{\ell}\right) \gamma\right)$ is a representation of $T$. Thus the rank of $T$ on $\mathbb{Q}$ is at most $n$.

Lemma 2. For any subset $P$ of $C$ such that $P \neq \emptyset$ and $P \neq C$, there is a $P$-augmenting word of length at most $2(n-1)$.

Proof. Since $\mathcal{A}$ is synchronized and irreducible, there is a synchronizing word $u$ such that $Q \cdot u$ is a single state $r$ belonging to $P$. Let $k$ be a positive integer such that $\mathrm{km} \geq \ell$, where $m$ is the length of the cycle $C$. We have $Q \cdot u a^{k m-\ell} a^{\ell}=r \cdot a^{k m}=r$. Hence $u a^{k m-\ell} a^{\ell}$ also is a synchronizing word focusing to $r$. Let $R$ denote the characteristic row vector of $r$. Since $q \cdot u=r$ for all $q \in Q$ and since $C$ has $m$ elements, we have $C M_{u}=m R$. Moreover, since $r \in C, R M_{a^{m}}=R$. We have

$$
\begin{aligned}
\left\langle T, u a^{k m-\ell}\right\rangle & =\left\langle S, u a^{k m}\right\rangle \\
& =C M_{u a^{k m}} P^{t}-C P^{t} \\
& =C M_{u} M_{a^{k m}} P^{t}-C P^{t} \\
& =m R M_{a^{k m}} P^{t}-C P^{t} \\
& =m R P^{t}-C P^{t} \\
& =m-\operatorname{card}(P) \neq 0 .
\end{aligned}
$$

As a consequence $T$ is non null.

Since $T$ has rank at most $n$ on $\mathbb{Q}$, there is a word $v$ of length at most $n-1$ such that $\langle T, v\rangle \neq 0$ (see [7, p. 145] or [15, p. 492 corollaire 4.19]).

If there is word $v$ of length at most $n-1$ such that $\langle T, v\rangle>0$, then $v a^{\ell}$ is a $P$-augmenting word and the claim is proved. Otherwise, there is a word $v$ of length at most $n-1$ such that $\langle T, v\rangle<0$.

Since $\ell$ is the level of $\mathcal{A}$, the vector $C M_{v a^{\ell}}$ is a linear combination of elements of $C$ and the sum of its coefficients is equal to $m$. 
We have

$$
\begin{aligned}
\sum_{i=0}^{m-1}\left\langle T, v a^{i}\right\rangle & =\sum_{i=0}^{m-1}\left\langle S, v a^{\ell} a^{i}\right\rangle=\sum_{i=0}^{m-1} C\left(M_{v a^{\ell} a^{i}}-I\right) P^{t} \\
& =\left(\sum_{i=0}^{m-1} C M_{v a^{\ell}} M_{a^{i}}-\sum_{i=0}^{m-1} C\right) P^{t}, \\
& =\left(C M_{v a^{\ell}}\left(\sum_{i=0}^{m-1} M_{a^{i}}\right)-m C\right) P^{t} \\
& =\left(\sum_{r \in C} r M_{v a^{\ell}} \sum_{i=0}^{m-1} M_{a^{i}}-m C\right) P^{t}, \\
& =\left(\sum_{r \in C} C-m C\right) P^{t}=0 .
\end{aligned}
$$

Indeed, for any $r$ in $C$, the state $q=r \cdot v a^{\ell}$ is in $C$ and for any state $q$ in $C$, the row of index $q$ of the matrix $\sum_{i=0}^{m-1} M_{a^{i}}$ is the row vector $C$.

As a consequence, there is a word $w$ of length at most $n+m-2$ such that $\langle T, w\rangle>0$. Hence there is a $P$-augmenting word of length at most $n+m-2+\ell$.

Thus, in all cases, there is a word of length at most $n+m-2+\ell$ which is $P$-augmenting.

To prove Proposition 2, we show that $\mathcal{A}$ has a synchronizing word of length at most $1+2 m(n-2)$. Indeed, let $P_{1}$ be reduced to an arbitrary state of $C$. If $P_{1}=C$ (that is to say if $m=1$ ), then $Q \cdot a^{\ell} \subseteq P_{1}$, and thus $a^{\ell}$ is a synchronizing word.

Otherwise, by Lemma 2 , there exists a word $u_{1}$ of length at most $n+m-2+\ell$ which is $P_{1}$-augmenting. Set $P_{2}=P_{1} u_{1}^{-1} \cap C$. If $P_{2} \neq C$, there is a word $u_{2}$ of length at most $n+m-2+\ell$ which is $P_{2}$-augmenting, and so on. In this way, we build a sequence $u_{1}, \ldots, u_{t-1}$ of words and a sequence $P_{1}, \ldots, P_{t}$ of sets of states, with $t \leq m$, such that, for $1 \leq i<t$,

- $u_{i}$ is a $P_{i}$-augmenting word of length at most $n+m-2+\ell$;

$-P_{i+1}=P_{i} u_{i}^{-1} \cap C$;

$-P_{t}=C$.

Then the word $a^{\ell} u_{t-1} \ldots u_{1}$ is a synchronizing word of length at most $\ell+(m-1)(n+m-2+\ell)$. Indeed, $Q \cdot a^{\ell} u_{t-1} \ldots u_{1} \subseteq C \cdot u_{t-1} \ldots u_{1} \subseteq$ $P_{t-1} \cdot u_{t-2} \ldots u_{1} \subseteq \ldots P_{2} \cdot u_{1} \subseteq P_{1}$. 
Since $m \leq n-\ell$ and $m \leq n-1$, we have

$$
\begin{aligned}
\ell+(m-1)(n+m+\ell-2) & \leq \ell+(m-1)(2 n-2) \\
& \leq n-m+2 m n-2 n-2 m+2 \\
& \leq 2 m n-n-3 m+2 \\
& =(n-2)(2 m-1)+m \\
& \leq 1+2 m(n-2) \leq 1+2(n-1)(n-2),
\end{aligned}
$$

which completes the proof.

\section{Application to finite prefix codes}

In this section we show how the previous result can be applied to the automaton associated to a finite prefix code.

A prefix code on the alphabet $A$ is a set $X$ of words on $A$ such that no element of $X$ is a prefix of another word of $X$.

A prefix code is maximal if it is not contained in another prefix code on the same alphabet. As an equivalent definition, a prefix code $X$ is maximal if for any word $u$ in $A^{*}$ has a prefix in $X$ or is a prefix of a word of $X$.

For a deterministic automaton $\mathcal{A}$ and an initial state $i$, the set $X_{\mathcal{A}}$ of labels of first return paths from $i$ to $i$ is a prefix code. If the automaton is complete, the prefix code is maximal.

Conversely, for any finite prefix code $X$, there exists a deterministic automaton $\mathcal{A}$ such that $X=X_{\mathcal{A}}$. Moreover, the automaton $\mathcal{A}$ can be supposed to be irreducible. If $X$ is a maximal prefix code, the automaton $\mathcal{A}$ is complete.

The automaton $\mathcal{A}$ can be chosen as follows. The set of states is the set $Q$ of prefixes of the words of $X$. The transitions are defined for $p \in Q$ and $a \in A$ by $p \cdot a=p a$ if $p a$ is a prefix of a word of $X$, and by $p \cdot a=\varepsilon$ if $p a \in X$. This automaton, denoted $\mathcal{A}_{X}$ is a decoder of $X$. Let indeed $\alpha$ be a one-to-one map from a source alphabet $B$ onto $X$. Let us add an output label to each edge of $\mathcal{A}_{X}$ in the following way. The output label of $(p, a, q)$ is $\varepsilon$ if $q \neq \varepsilon$ and is equal to $\alpha^{-1}(p a)$ if $q=\varepsilon$. With this definition,

for any word $x \in X^{*}$, the output label of the path $i \stackrel{x}{\rightarrow} i$ is the word $\alpha^{-1}(x)$.

Let us show that, as a consequence of the fact that $X$ is finite, the automaton $\mathcal{A}$ is additionally one-cluster with respect to any letter.

Indeed, let $a$ be a letter and let $C$ be the set of states of the form $i \cdot a^{j}$. For any state $q$, there exists a path $i \stackrel{u}{\rightarrow} q \stackrel{v}{\rightarrow} i$. We may suppose that $i$ 
does not occur elsewhere on this path. Thus $u v \in X$. Since $X$ is a finite maximal code, there is an integer $j$ such that $u a^{j} \in X$. Then $q \cdot a^{j}=i$ belongs to $C$. This shows that $\mathcal{A}$ is one-cluster with respect to $a$.

A maximal prefix code $X$ is synchronized if there is a word $x \in X^{*}$ such that for any word $w \in A^{*}$, one has $w x \in X^{*}$. Such a word $x$ is called a synchronizing word for $X$.

Let $X$ be a synchronized prefix code. Let $\mathcal{A}$ be an irreducible deterministic automaton with an initial state $i$ such that $X_{\mathcal{A}}=X$. The automaton $\mathcal{A}$ is synchronized. Indeed, let $x$ be a synchronizing word for $X$. Let $q$ be a state of $\mathcal{A}$. Since $\mathcal{A}$ is irreducible, there is a path $i \stackrel{u}{\rightarrow} q$ for some $u \in A^{*}$. Since $x$ is synchronizing for $X$, we have $u x \in X^{*}$, and thus $q \cdot x=i$. This shows that $x$ is a synchronizing word for $\mathcal{A}$.

Conversely, let $\mathcal{A}$ be an irreducible complete deterministic automaton. If $\mathcal{A}$ is a synchronized automaton, the prefix code $X_{\mathcal{A}}$ is synchronized. Indeed, let $x$ be a synchronizing word for $A$. We may assume that $q \cdot x=i$ for any state $q$. Then $x$ is a synchronizing word for $X$.

Proposition 3. Let $X$ be a maximal synchronized prefix code with $n$ codewords on an alphabet of size $k$. The decoder of $X$ has a synchronizing word of length at most $O\left((k n)^{2}\right)$.

Proof. The automaton $\mathcal{A}_{X}$ is one-cluster. The number $N$ of its states is the number of prefixes of the words of $X$. Thus $N=(n-1)(k-1)$ since a complete $k$-ary tree with $n$ leaves has $(n-1)(k-1)$ internal nodes. By Proposition 2, there is a synchronizing word of length at most $1+2(N-1)(N-2)$, whence $O\left((k n)^{2}\right)$.

Example 1. Let us consider the following Huffman code $X=(00+01+$ $1)(0+10+11)$ corresponding to a source alphabet $B=\{a, b, c, d, e, f, g, h, i\}$ with a probability distribution $(1 / 16,1 / 16,1 / 8,1 / 16,1 / 16,1 / 8,1 / 8,1 / 8$, $1 / 4)$. The Huffman tree is pictured in the left part of Figure 1 while the decoder automaton $\mathcal{A}_{X}$ is given in its right part. The word 010 is a synchronizing word of $\mathcal{A}_{X}$.

When the lengths of the codewords in $X$ are not relatively prime, the automaton $\mathcal{A}_{X}$ is never synchronized (see Example of Figure 1). When the lengths of the codewords in $X$ are relatively prime, the code $X$ is not necessarily synchronized. However, there is always another Huffman code $X^{\prime}$ corresponding to the same length distribution which is synchronized by a result of Schützenberger [16]. One can even choose $X^{\prime}$ such that the underlying graph of $\mathcal{A}_{X}$ and $\mathcal{A}_{X^{\prime}}$ are the same. This is a particular case of the road coloring theorem of due to Trahtman [18] (see also [2]). The 

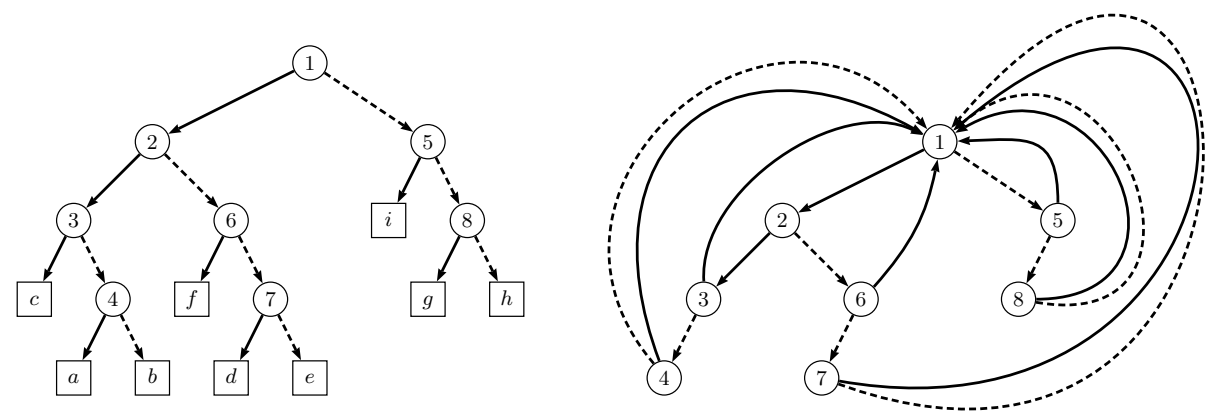

Fig. 2. A synchronized Huffman code $X$ on the left and its decoder $\mathcal{A}_{X}$ on the right.

particular case corresponding to finite prefix codes was proved before in [11].

Our result guarantees that the Huffman decoder has a synchronizing word of length at most quadratic in the number of nodes of the Huffman tree.
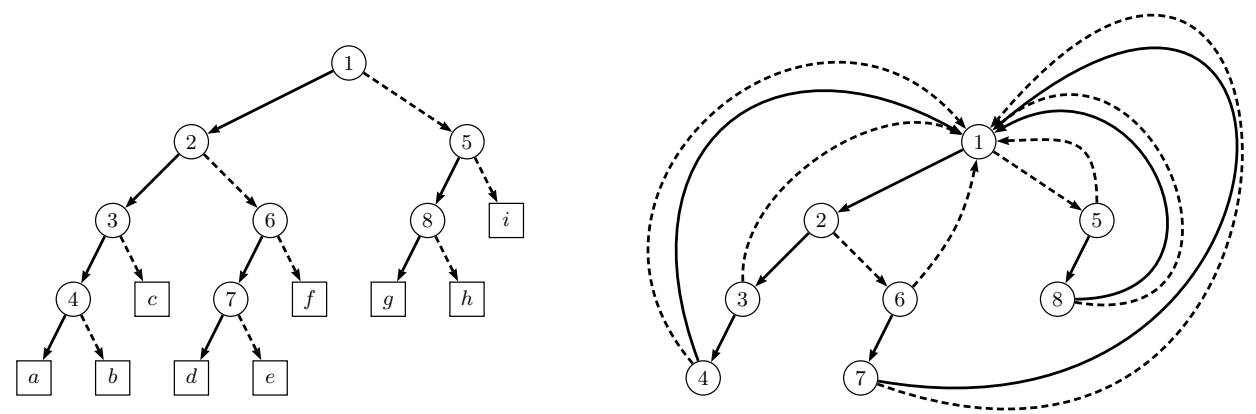

Fig. 3. A non synchronized Huffman code $X$ on the left and its decoder on the right. The automaton on the right is not synchronized. Indeed, for any word $w$, the set of states reachable by $w$ is either $\{1,3\},\{2,4\},\{1,5\}$, or $\{1,6\}$.

\section{References}

1. M.-P. BÉAL, A note on Černý's conjecture and rational series. preprint IGM 
2003-05. Unpublished, 2003.

2. M.-P. BÉAL AND D. PERRIN, A quadratic algorithm for road coloring, CoRR, abs/0803.0726 (2008).

3. M. T. BISKUP, Shortest synchronizing strings for Huffman codes, in MFCS, 2008, pp. $120-131$.

4. A. CARPi And F. D'Alessandro, The synchronization problem for strongly transitive automata, in Developments in Language Theory, 2008, pp. 240-251.

5. J. ČERnÝ, Poznámka $k$. homogénnym experimentom s konecnými automatmi, Mat. fyz. čas SAV, 14 (1964), pp. 208-215.

6. L. Dubuc, Sur les automates circulaires et la conjecture de Černý, RAIRO Inform. Théor. Appl., 32 (1998), pp. 21-34.

7. S. Eilenberg, Automata, languages, and machines. Vol. A, Academic Press [A subsidiary of Harcourt Brace Jovanovich, Publishers], New York, 1974. Pure and Applied Mathematics, Vol. 58.

8. C. F. Freiling, D. S. Jungreis, F. Théberge, and K. Zeger, Almost all complete binary prefix codes have a self-synchronizing string, IEEE Transactions on Information Theory, 49 (2003), pp. 2219-2225.

9. J. KARI, A counter example to a conjecture concerning synchronizing words in finite automata, EATCS Bulletin, 73 (2001), p. 146.

10. — Synchronizing finite automata on Eulerian digraphs, in MFCS'2001, no. 2136 in LNCS, Berlin, 2001, Springer, pp. 432-438.

11. D. PERrin AND M.-P. SChütZEnBERGER, Synchronizing words and automata and the road coloring problem, in Symbolic Dynamics and its Applications, P. Walters, ed., American Mathematical Society, 1992, pp. 295-318. Contemporary Mathematics, vol. 135 .

12. J.-E. PIN, Le problème de la synchronisation et la conjecture de Černý, thèse de 3ème cycle, Université Paris VI, 1978.

13. _ Sur un cas particulier de la conjecture de Černý, in 5th ICALP, no. 62 in LNCS, Berlin, 1978, Springer, pp. 345-352.

14. - On two combinatorial problems arising from automata theory, Annals of Discrete Mathematics, 17 (1983), pp. 535-548.

15. J. SAKArovitch, Éléments de théorie des automates, Éditions Vuibert, 2003.

16. M.-P. Schützenberger, On synchronizing prefix codes, Inform. and Control, 11 (1967), pp. 396-401.

17. A. N. Trahtman, Synchronization of some DFA, in TAMC, 2007, pp. 234-243.

18. A. N. Trahtman, The road coloring problem, Israel J. Math., (2008). to appear.

19. A. Trakhtman, Some aspects of synchronization of DFA, J. Comput. Sci. Technol., 23 (2008), pp. 719-727.

20. M. V. VOLKOV, Synchronizing automata preserving a chain of partial orders, in CIAA, 2007, pp. 27-37.

21. _ Synchronizing automata and the Černy conjecture, in LATA, 2008, pp. 1127. 